\title{
Clinicopathologic study of breast lumps in Abakaliki, South Eastern Nigeria
}

\author{
Ogbuanya Aloysius Ugwu-Olisa', Anyanwu Stanley Nnamdi C², Nwigwe Chinedu Gregory'1, lyare Festus $E^{3}$ \\ ${ }^{1}$ Department of surgery, Federal Teaching Hospital, Abakaliki (FETHA), Ebonyi State, Nigeria, ${ }^{2}$ Department of surgery, Nnamdi \\ Azikiwe University Teaching Hospital, Nnewi, Anambra State, Nigeria, ${ }^{3}$ Department of Pathology, Federal Teaching Hospital, Abakaliki, \\ Ebonyi State
}

Aims and Objectives: Breast lumps are quite common, but the principal issue is differentiating malignant from the benign sub-groups. The determinants of histologic diagnosis are multifactorial, ranging from environmental, genetic to sex and age-related factors. This study aimed to document the spectrum of breast lumps in our environment. Materials and Methods: This is a descriptive prospective analysis of consecutive breast lumps biopsied at Federal Teaching Hospital Abakaliki, between December 2010 to November 2012. The data were analysed using statistical package for social sciences (SPSS) version 22.0. Results: A total of 302 patients with 359 breast lumps were seen, 298 females and 4 males giving male:Female ratio of $1: 75$. The ages of the patients ranged between $16-82$ years with a mean of $37.0+/$ - SD 13.0. Majority (56.3\%) of the patients had benign lumps. Fibroadenoma was the commonest $(33.8 \%)$, followed closely by invasive ductal carcinoma $(32.8 \%)$, then fibrocystic change $(17.2 \%)$. The occurrence of breast cancer was strongly age-related, being 1 in 5 biopsies at 20-29 years and 3 in 5 biopsies at 40-49 years age groups. Over two-third $(69.0 \%)$ of 132 patients with breast cancers were premenopausal. Majority $(78.8 \%)$ of the 132 patients with breast cancer presented with advanced disease. At a median follow up of 9 months, $13(9.8 \%)$ and $6(4.5 \%)$ of 132 cancer patients developed recurrences and new cancer sites respectively. Conclusion: Most patients with breast complaints present with lumps and majority are benign, fibroadenoma being the commonest histology. Occurrence of breast cancer is strongly age-related with majority of cancer patients presenting late.

\section{Access this article online}

Website:

http://nepjol.info/index.php/AJMS DOI: 10.3126/ajms.v7i3.13772 E-ISSN: 2091-0576 P-ISSN: $2467-9100$

Key words: Breast, Clinicopathology, Lumps, Southeast

\section{INTRODUCTION}

Globally, breast diseases are showing a rising trend and as a result, there is considerable interest in the clinicopathologic studies of breast masses in various populations. ${ }^{1,2}$ In recent decades, gains of life expectancy from industrialization have changed previous trends of disease in developing nations, creating a shift from diseases of pestilence and infections to those of cancers, trauma, cardiovascular disease and mental illness. ${ }^{3,4}$ Regional heterogeneity highlights the importance of understanding local burden of diseases and setting goals and targets, taking such patterns into account. ${ }^{3}$ Published data indicate that the rate of diagnosis of breast diseases has increased worldwide due to recent increase in awareness campaigns, greater availability of diagnostic and screening facilities and establishment of dedicated breast clinics. ${ }^{5-8}$
The finding of a breast change in a woman's breast is usually accompanied by inordinate anxiety and reactions from the patient and her family. ${ }^{7,8}$ Because such change has a variable spectrum of presentations and pathologic backgrounds, modern acceptable practice requires a step-wise diagnostic approach involving clinical, radiological and pathological assessments. ${ }^{5,7}$ In our setting like many other resource-poor communities in the developing world, the availability and expertise for mammography component of the triple test is limited. ${ }^{9}$ Nevertheless, studies have shown that combined diagnostic information from clinical and pathological evaluation yields satisfactory diagnostic accuracy. ${ }^{5,10}$

The vast majority of referred patients with breast disorders present with breast lumps and receive benign diagnosis when subjected to histopathology examination. ${ }^{7,8,10-12}$ 
Results from many published studies reveal that breast cancer is the leading female malignancy in the world and is now the commonest cancer in Nigeria. ${ }^{4,6}$ It is against this background that the usual management of a palpable breast mass is based on the assumption that the mass is cancerous until proven otherwise. ${ }^{8}$ The need for proper evaluation of breast lumps is further emphasized by the occurrence of a subset of benign breast lesions categorized as proliferative breast diseases. ${ }^{8,13-15}$ These variants confer increased risk of progression to malignancy and their histologic diagnosis mandates mammographic surveillance even after excision. ${ }^{8,10,13-15}$ Similarly, the relative risk of invasive breast cancer is higher in women with complex fibroadenomas and remained elevated for decades after diagnosis. ${ }^{14,15}$ This risk further increases in patients with concomitant family history of breast cancer. ${ }^{14,15}$

Data on breast diseases are scanty in our setting despite the recent global clamor for increased awareness campaigns in different populations. ${ }^{1,8}$ Available data generated from similar surveys on breast diseases from other populations provided formidable opportunities for knowledge of the disease spectrum, counseling, intervention strategies, advocacy for breast health programs and screening for early detection of breast cancers. ${ }^{16}$ The aim of this study is to analyse the spectrum of presentations and pathology of breast lumps at Federal Teaching Hospital Abakaliki, South eastern Nigeria.

\section{MATERIALS AND METHODS}

\section{Design and settings}

This is a descriptive prospective study of patients with palpable breast lumps seen at Federal Teaching Hospital, Abakaliki (FETHA) between December 2010 to November 2012. FETHA is a teaching health institution located in the southeastern region of Nigeria.

\section{Patients}

All consecutive 302 patients with histology reports of breast biopsies were included. Patients were required to give written informed consent prior to enrolment in this study. Patients without discretely palpable breast lumps and those who failed to give consent for biopsies were excluded. All the patients were seen and interviewed by either a general surgeon or senior registrar in general surgery at the surgical outpatient clinic of the hospital. Records of socio-demographic and relevant clinical data of each patient were obtained and entered into a standard proforma. Clinical diagnosis of benign or malignant breast lump after comprehensive history and physical examination was made. Radiological evaluation was not routinely done because of lack of dedicated ultrasound and mammographic machines in our breast clinic.
Basic investigations like haemoglobin level and urinalysis were routinely requested and their results recorded. Focused tests like chest and spine $\mathrm{x}$-rays, abdominopelvic ultrasound and liver function tests were reserved for those with clinically suspicious malignant lesions or co-morbidities. The method of biopsy used was either open (excision and incision) or trucut needle biopsies depending on size and clinical parameters at presentation. All the biopsies were carried out by either a consultant general surgeon or a senior registrar in general surgery. Majority of the patients had their biopsies under local infiltrative anesthesia using $5-20 \mathrm{ml}$ of $0.5 \%$ xylocaine in double dilution. Fewer patients required general anesthesia.

Circumareolar, followed by circumferential incisions were mostly used for oncoplastic purposes. Diathermy dissection was adopted in majority of cases to enhance haemostasis. Combined sharp and blunt dissection was used for tissue retrieval. For excision biopsies, passive drains were inserted in few cases with extensive dissection. Majority of skin incisions were closed with subcuticular stitches using vicry $2 / 0$; others were executed with nylon $2 / 0$ interrupted suturing.

The resection specimens were preserved with $10 \%$ formal saline, labeled and sent to histopathology laboratory accompanied with detailed and completed histology form. Majority of the biopsies were performed on day case basis. One pathologist reported all the slides. Follow up visits were arranged and the results of histology subsequently entered into the proforma usually 2-4 weeks from the time of biopsy. Available patients were followed up for two years.

\section{Statistical data analysis}

Statistical data analysis was done using statistical package for social sciences (SPSS) software version 22.0 (IBM, USA 2015). Descriptive statistics were employed to calculate categorical variables like percentages. Mean, median and standard deviation were used to summarize continuous variables. Chi-square test was used to determine the $\mathrm{P}$-values for categorical variables, with a P-value of less than 0.05 considered to constitute a statistically significant difference.

\section{Ethical consideration}

The protocol for the study was approved by the Research and Ethical committee of the Federal Teaching Hospital, Abakaliki, before commencement of the study.

\section{RESULTS}

A total of 302 patients with biopsy-confirmed breast lumps were enrolled. This figure represents $80.5 \%$ of the entire 
375 patients with breast complaints seen at our surgical clinic over a period of two years. The ages of the patients ranged from 16-82 years with a mean of 37.0 +/- SD 13.0. The age distribution of the patients seen during the study period is shown in Table 1 below.

There were 298 females and 4 males giving a male to female ratio of 1:75. Among female patients, 169 (56.7\%) had benign lumps, the remaining 129 (43.3\%) harbored malignant tumors. Three of the four male patients had malignancy, only one had benign diagnosis. Overall, the male to female ratios for benign and malignant lumps were 1:169 and 1:43 respectively. Of the 298 females, 98 (32.9\%) were nulliparous, the remaining $200(67.1 \%)$ had parity that ranged from 1-12. Out of 162 multiparous women (parity of 3-12), 105 (64.8\%) had malignant histology, the rest received benign histologic diagnoses.

Over two third $(89,69.0 \%)$ of the 129 women with malignant biopsies were premenopausal, nearly a quarter $(32,24.8 \%)$ were postmenopausal and the remaining $8(6.2 \%)$ were peri-menopausal. Majority $(150,88.8 \%)$ of the 169 women with benign lumps were also premenopausal, 18(10.6\%) postmenopausal while only $1(0.6 \%)$ was premenstrual.

The histopathology diagnoses of breast lumps studied are shown in Table 2 below.

A total of $170(56.3 \%)$ out of 302 patients examined had benign lumps; the remaining 132 (43.7\%) patients harbored malignant lumps. Seven (2.3\%) patients (all premenopausal) had histology diagnosis of proliferative type of fibrocystic disease of the breast. Male breast cancer constituted 2.3\% of the 132 patients with breast cancer, but mammary disease of males accounted for only $1.3 \%$ of the entire 302 patients with breast lumps in this study. The sitespecific intra-mammary distribution of the breast lumps is shown in Figure 1 below.

Most $(277,91.7 \%)$ patients had their biopsies under local anesthesia, the remaining $25(8.3 \%)$ were operated under general anesthesia.
A total of 359 lumps in 302 patients were evaluated. On the right side, 164(45.7\%) lumps were recorded; 136 were solitary (from 136 patients) while 28 were multiple from 12 individuals giving a total of 148 patients. Similarly, $140(39.0 \%)$ lumps were encountered on the left side, 118 were solitary (from 118 patients) while 22 were multiple from nine patients giving a total of 127 patients. Additionally, 55(15.3\%) lumps from 27 patients with bilateral disease were recorded. This gives bilateral to unilateral ratio of 1:10 for patients with breast lumps. No patients with multiple or bilateral lumps had dual histopathologic diagnosis.

The duration of symptoms before presentation ranged from 4 days to 15 years with a mean of $2.8+/$ - SD 2.3 . Approximately one third $(103,34.1 \%)$ presented within 3 months of onset, with less than a quarter $(68,22.5 \%)$ presenting within one month of illness. Furthermore, $47(15.6 \%), 32(10.6 \%)$ and $120(39.7 \%)$ patients presented at 3-6 months, 6-12 months and beyond one year of onset respectively. Thirty four (11.3\%) patients discovered their lumps by breast self examination (BSE), 258 (85.4\%) through accidental discovery, $8(2.6 \%)$ by their spouses; lumps in the remaining 2 patients were discovered by their doctors. Other clinicopathological features of breast lumps evaluated in this study are shown in Table 3 below.

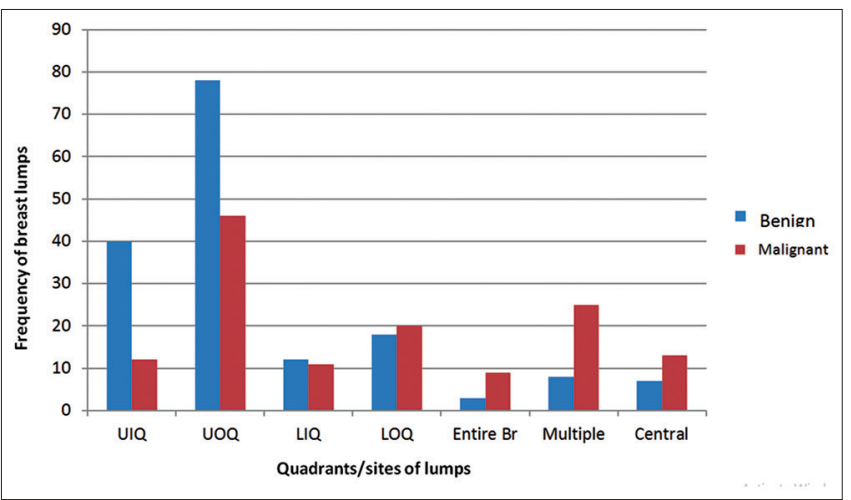

Figure 1: Distribution of breast lumps in the various quadrants/sites. $\mathrm{UIQ}=$ Upper inner quadrant; $\mathrm{UOQ}=$ Upper outer quadrant; $\mathrm{LIQ}=$ Lower inner quadrant. $\mathrm{LOQ}=$ Lower outer quadrant; Entire $\mathrm{Br}=$ Entire breast; Multiple=Multiple quadrants; Central=Central area

\section{Table 1: Correlation of histopathology and age}

\begin{tabular}{|c|c|c|c|c|c|c|}
\hline Age range (years) & Fibro-adenoma & Fibrocystic disease & Cancer & Phyllodes tumor & Others & Total (\%) \\
\hline $10-19$ & 29 & 4 & 0 & 0 & 2 & $35(11.6)$ \\
\hline $20-29$ & 44 & 10 & 14 & 1 & 3 & $72(23.8)$ \\
\hline $30-39$ & 15 & 18 & 29 & 1 & 4 & $67(22.2)$ \\
\hline $40-49$ & 13 & 15 & 37 & 2 & 2 & 69 (22.9) \\
\hline $50-59$ & 1 & 3 & 35 & 1 & 0 & $40(13.2)$ \\
\hline $60-69$ & 0 & 2 & 10 & 0 & 0 & $12(4.0)$ \\
\hline $70-79$ & 0 & 0 & 4 & 0 & 0 & $4(1.3)$ \\
\hline $80-89$ & 0 & 0 & 3 & 0 & 0 & $3(1.0)$ \\
\hline Total (\%) & $102(33.8)$ & $52(17.2)$ & $132(43.7)$ & $5(1.7)$ & $11(3.6)$ & $302(100.0)$ \\
\hline
\end{tabular}


Among 132 breast cancer patients, clinical staging showed that $2(1.5 \%)$ were in stage I, $26(19.7 \%)$ in stage II, $63(47.7 \%)$ in stage III and $41(31.1 \%)$ in stage IV. The ages of patients with breast cancer ranged from $21-82$ years, peaks at 40-49 years with a mean of $46.1+/$ - SD 13.5. Five (3.8\%) patients with breast cancer were below 25 years old. Similarly, ages of patient with fibroadenoma ranged from 16-52 years with a mean of $26.0+/-$ SD 10.0 , while that of fibrocystic disease ranged from 19-67 years with a mean of $36.2+/$-SD 11.6.

Out of 302 patients studied, 251(83.1\%), 201(66.6\%) and $148(49.0 \%)$ were available for follow up at 1 month, 3 months and 6 months respectively. Less than half, $133(44.0 \%)$ were available at one year while approximately one third $(103,34.1 \%)$ were followed up till two years. The duration of follow up ranged from 1-24 months with a median of 9 months. Among the follow up cancer patients,

\begin{tabular}{lcc} 
Table 2: Histopathological types of breast lumps \\
\hline Histology diagnosis & Frequency & Percentage \\
\hline Fibroadenoma & 102 & 33.8 \\
Fibrocystic disease & 52 & 17.2 \\
Benign phyllodes tumor & 5 & 1.7 \\
Duct ectasia & 3 & 1.0 \\
Chronic mastitis & 2 & 0.7 \\
Granula cell tumor & 1 & 0.3 \\
Reactive intramammary lymph node & 1 & 0.3 \\
Neurofibroma & 1 & 0.3 \\
Gynaecomastia & 1 & 0.3 \\
Lactating adenoma & 1 & 0.3 \\
Lipoma & 1 & 0.3 \\
Invasive ductal carcinoma & 99 & 32.8 \\
Invasive lobular carcinoma & 16 & 5.3 \\
Medullary carcinoma & 6 & 2.0 \\
Ductal carcinom in-situ (DCIS) & 2 & 0.7 \\
Papillary cancer & 3 & 1.0 \\
Mucinous cancer & 4 & 1.3 \\
Burkittis lymphoma & 2 & 0.7 \\
Total & 302 & 100.0 \\
\hline
\end{tabular}

13(9.8\%) patients developed cancer recurrences, $6(4.5 \%)$ patients developed new cancer sites at the contralateral breasts after 18 months of follow up. A 36 years old woman with biopsy-confirmed fibrocystic disease developed invasive ductal carcinoma at 23 months of follow up. Four $(3.0 \%)$ breast cancer patients died in the hospital during follow up, though $41(31.1 \%)$ patients with breast cancer were lost from follow up after one year of surveillance.

\section{DISCUSSION}

Heightened anxiety associated with breast cancer diagnosis causes many patients with breast disease to attend specialist surgical clinics irrespective of their ages, sex or presenting complaints. ${ }^{78,17}$ Majority of patients with breast disease at our locality within the study period are relatively young females presenting with palpable lumps and having a preponderance of benign disease. This breast disease pattern conforms with reports from other populations. ${ }^{8,10,12,17}$ The percentage of benign breast disease $(56.3 \%)$ recorded in this study is in common agreement with a figure of $58.8 \%$ reported in Sokoto, ${ }^{18}$ Nigeria. Reports from similar studies ${ }^{10,12,19}$ in Ibadan $(89.4 \%$ ) Nigeria, India $(92.6 \%)$ and Ghana $(80.9 \%)$ showed preponderance of benign histology and support the above pattern, with a caveat that their rates of benign biopsies were higher than ours. These variations are expected because the studies were not conducted in the same age groups, races or study protocols.

The growing rates of benign breast disease in the developing communities of the world ${ }^{8,17}$ have important epidemiological and clinical implications. Reports culled from western literature implicate premenopausal status, family history of breast cancer, delayed age at first live birth ( $>25$ years) and low parity with increased risk of progression to invasive cancer in women biopsied for benign breast disease. ${ }^{13-15} \mathrm{In}$

\begin{tabular}{|c|c|c|c|c|c|}
\hline Clinical/path parameters & Fibroadenoma & Fibrocystic change & Cancer & Others & Total $(\%)$ \\
\hline \multicolumn{6}{|l|}{ Complaints } \\
\hline Lump alone & 99 & 22 & 113 & 15 & $249(82.4)$ \\
\hline Lump+pain & 3 & 28 & 4 & 1 & $36(11.9)$ \\
\hline Ulcer+pain & 0 & 0 & 15 & 0 & $15(5.0)$ \\
\hline Pain alone & 0 & 2 & 0 & 0 & $2(0.7)$ \\
\hline \multicolumn{6}{|l|}{ Biopsy type } \\
\hline Excision & 98 & 49 & 18 & 15 & $180(59.6)$ \\
\hline Incision & 1 & 2 & 47 & 1 & 51 (16.9) \\
\hline Trucut & 3 & 1 & 67 & 0 & $71(23.5)$ \\
\hline \multicolumn{6}{|l|}{ Size $(\mathrm{cm})$} \\
\hline$\leq 5$ & 98 & 50 & 22 & 12 & $182(60.3)$ \\
\hline$>5$ & 4 & 2 & 110 & 4 & $120(39.7)$ \\
\hline \multicolumn{6}{|l|}{ Axillary node } \\
\hline Present & 1 & 0 & 118 & 3 & $122(40.4)$ \\
\hline Absent & 101 & 52 & 14 & 13 & $180(59.6)$ \\
\hline
\end{tabular}


agreement with reported series in Africa, ${ }^{7,8,20}$ Yemen $^{21}$ and Pakistan, ${ }^{11}$ we found that majority $(88.2 \%)$ of women with benign lumps are premenopausal, expressing yet greater concern for invasive cancer risk in young women in these populations. The discovery of seven $(2.3 \%)$ premenopausal women with proliferative breast disease in this series further increases curiosity for higher cancer risk in young women with specific benign breast lumps. These observations possibly suggest the etiologic role of some benign lumps in the increasing incidence and occurrence of breast cancer in younger women in developing nations compared to their white counterparts.

The histologic types of breast cancer reported in this study conform with data from previous studies in Africa, ${ }^{7,8,10,22}$ Pakistan ${ }^{2,11}$ and India. ${ }^{19}$ The frequency of invasive ductal cancer among cancer cases in this study $(75.0 \%)$ is in consonance with figures documented in Sokoto ${ }^{18}(76.2 \%)$, Nigeria and Yemen ${ }^{21}(86.6 \%)$. Similar results were noted by Ellis ${ }^{23}$ from a referral hospital in London. This perhaps highlights the fact that the differences in the clinical profile of breast cancer in Africa and western economies are not due to histologic types, but may be related to tumor biology, delay in presentation, poverty and socio-cultural practices. Anyanwu ${ }^{6}$ and Akande and Colleagues ${ }^{9}$ both in Nigeria share similar views. Indeed, evidence from Africa on the emerging breast cancer epidemic in the continent shows that the current beliefs about hormone receptor subtypes of breast cancer in Africa is probably not systematically different from the pattern in other populations after adjusting for factors like age and that the reported differences are related to poor tissue handling and laboratory processing practices. ${ }^{4}$ Overall, breast cancer (43.7\%) accounted for significant proportion of breast biopsies in this study, similar to a figure of $37.2 \%$ in Maiduguri, ${ }^{22}$ Nigeria. Of note, breast cancer is commoner than benign lesion in Nnewi ${ }^{7}$ Nigeria, Pakistan ${ }^{2}$ and London $^{23}$ where cancer preponderance was ascribed to selective referral of cancer cases to tertiary hospitals like ours while benign lumps are generally managed in private hospitals.

Previous studies on awareness and practice of breast selfexamination in Abakaliki revealed that while the level of awareness of breast cancer is high among nurses (93.7\%) and market women $(77.7 \%$ ), only $38.9 \%, 13.0 \%$ and $13.4 \%$ of the 238 market women were aware of Breast Self Examination (BSE), Clinical Breast Examination (CBE) and mammography respectively as methods of early detection of breast cancer. ${ }^{24}$ Worse still, the study showed that only $23.9 \%$ of the market women had knowledge of how to do Breast Self Examination (BSE), 21.8\% have practiced it in the past while a negligible $0.4 \%$ (a woman) knew the correct frequency of BSE and does it regularly. ${ }^{24}$ In sum, Abakaliki is still underserved with breast cancer awareness campaigns underscored by the high advanced breast cancer rate $(78.8 \%$ of 132 cancer cases) recorded in this study.

The age distribution of the patients in this study bears relationship with the histologic types. There was no malignancy detected in the under 20 years, in agreement with findings from Ibadan, ${ }^{12}$ Enugu $^{8}$ both in Nigeria, Ghana ${ }^{10}$ and Yemen. ${ }^{21}$ In Benin city, ${ }^{25}$ Nigeria, a single case of breast cancer was reported from 84 adolescents aged 10-19 years over a 10 year period. The pool of benign biopsies in that population ${ }^{25}$ of referred patients selected for age emphasizes the relative rarity of breast cancer in the young. Similar pattern was reported in Maiduguri, ${ }^{22}$ Nigeria. A rising trend of cancer incidence as the age increases was observed. At 20-29 years age group, malignancy occurred at the rate of 1 in 5 biopsies, rising to 2 in 5 biopsies at 30-39 years group $(\mathrm{p}<0.05)$. The increase in malignant diagnosis continued at 3 in 5 biopsies in 40-49 years group and in the 50-59 years and 60-69 years groups, it hits 4 in 5 biopsies before becoming total (all malignant) after 70 years of age $(p<0.05)$. This pattern is similar to findings in Ibadan, ${ }^{12}$ Nigeria and Ghana. ${ }^{10}$ The concentration of breast cancer cases in the relatively young patients (30-39, 40-49 and 50-59 years) in this and other studies ${ }^{10,12,18,22}$ in the developing nations is worrisome, but support previous reports ${ }^{6,9}$ that breast cancer occurs a decade earlier in black women than their white counterparts. This observation supports the rationale to commence breast cancer screening at 40 years of age or earlier in those with high risk factors.

Our finding that fibroadenoma is the single most common histological variant of breast lumps in this study is in common agreement with previous data. ${ }^{8,11,12,18,21}$ The predilection of Negroes to fibroadenoma has been previously reported. ${ }^{8,20}$ Elsewhere, genetic, environmental and socio-cultural factors have been implicated. ${ }^{8,20}$ In the current report, the single case of fibroadenoma with axillary lymph node due to native intervention underscores the usefulness of triple test assessment in all cases of breast lumps.

Fibrocystic disease, the second most common benign lump in this and other African studies ${ }^{7,8,12}$ is clinically important because of its high frequency and premalignant potential. $.10,17$ On a happier note, the non-proliferative variants are far more common than the proliferative types that confer increased risk of breast cancer. ${ }^{13,15,17}$ The percentage of proliferative pathology in this study $(2.3 \%)$ conforms with the rate of $2.7 \%$ in Benin City ${ }^{20}$ Nigeria, but lower than $13.5 \%$ in Jamaica, ${ }^{17}$ an Afro-Caribbean population with mixed races of African and Caucasian background.

The rarity of the minor breast tumors recorded in this study (phyllodes tumor, duct ectasia, gynaecomastia) 
has previously been reported and agrees with data from Maiduguri, ${ }^{22}$ Nnewi ${ }^{7}$ both in Nigeria and Ghana. ${ }^{10}$ Two striking observations were made with these uncommon breast tumors. A single case of duct ectasia with axillary lymph nodes was misdiagnosed as cancer by clinical and ultrasound methods, but histology confirmed its benignity. Second, a benign phyllodes tumor misdiagnosed as fibroadenoma even after trucut biopsy was correctly typed after histologic examination of the entire resected specimen. The clinical value of these observations lies on the differences in their surgical management and expresses the imperativeness of step-wise diagnostic approach for dominant breast masses.

\section{CONCLUSION}

The occurrence of different breast lumps in our environment has strong links with the ages and menstrual status of the patients. The benign lumps are commoner in younger premenopausal patients while breast cancer incidence increases with age and peaks around 40-49 years group. Enormous concern over cancer risk for the proliferative benign lesions exists since regular mammographic screening programme is still in abeyance in our setting. Breast cancer campaign in our environment remains inadequate because majority of the patients with breast cancer in this study presented with advanced disease. The need for a dynamic national program on breast cancer awareness campaign and screening is salutary if any meaningful outcomes are desired.

Source of support

None declared

\section{Conflict of interest}

None declared

\section{ACKNOWLEDGEMENT}

We are highly indebted to the head, department of surgery, Dr Okorie CC and all the consultant surgeons in our department for their understanding and kind cooperation during this study. We are grateful to all the residents of surgery department for their active involvement in data collection. We sincerely appreciate the nursing staff of the surgical outpatient clinic and members of staff of histopathology department and other members of staff of department of surgery FETHA for their understanding during this study.

\section{REFERENCES}

1. Jeje EA, Mofikoya BO and Oku YE. Pattern of breast masses in Lagos: a private health facility view of 189 consecutive patients. Nig Q J Hospital Med 2010; 20(1):38-41.
2. Siddiqui MS, Kayani N, Gill MS, Pervez S, Muzaffar S, Azziz SA, et al. Breast diseases: a histopathological analysis of 3279 cases at a tertiary care centre in Parkistan. J Park Med Assoc 2003; 53:94-97.

3. Thomas GW, Scott ER, Katherine DT, Alex BH, Stuart RL, William RB, et al. An estimation of the Global Volume of Surgery: a modeling strategy based on available data. Lancet 2008; 372(9633):139-144.

4. Akarolo-Anthony SN, Ogundiran TO and Adebamowo CA. Emerging Breast Cancer epidermic: evidence from Africa. Breast Cancer Res 2010; 12 (suppl 4): S8.

5. Tong FL. The role of Fine needle Aspiration cytology and Needle Core Biopsy in the diagnosis and management of breast cancers. Cytopathology 2007; 1(6); 8-12.

6. Anyanwu SNC. Temporal trend in Breast Cancer presentation in the third world. Journal of Experimental and Clinical Cancer Research 2008;27:17.

7. Egwuonwu OA, Anyanwu SNC, Chianakwana GU and Ihekwoaba EC. Breast Lumps in Nnamdi Azikiwe University Teaching Hospital, Nnewi, Nigeria: A 5 year review. Nigerian Journal of Surgery 2009; 15(2):6-9.

8. Njeze GE. Breast Lumps: A 21-year single centre Clinical and Histological Analysis. Nigerian Journal of Surgery 2014; 20: 38-41.

9. Akande HJ, Oyinloye OI and Olafimihan BB. Radiological findings of breast cancer screening in a newly equipped centre. International Journal of Medicine and Medical Sciences 2011;3(9):294-298.

10. Ohene-Yeboah MOK. An audit of excised breast lumps in Ghanaian women. WAJM 2005; 24(3): 252-255.

11. Aslam HM, Saleem S, Shaikh HA, Shahid N and Mughal A. Clinicopathological Profile of patients with breast diseases. Diagnostic Pathology 2013; 8:77.

12. Irabor DO and Okolo CA. An audit of 149 consecutive breast biopsies in Ibadan, Nigeria. Park J Med Sci 2008; 24(2):257-262.

13. Hartman LC, Sellers TA, Frost MH, Lingle WH, Degnim AC, Ghosh K, et al. Benign breast cancer and the risk of Breast cancer. New England J Med 2005; 353:229-237.

14. Guray $M$ and Sabin AA. Benign Breast Diseases: Classification, Diagnosis and management. The Oncologist 2006; 11:433-449.

15. Dorjgocho T, Deming SL, Gao YT, Lu N, Zheng Y, Ruan Z, et al. History of Benign Breast Disease and risk of breast Cancer among women in China; a case controlled study. Cancer Causes Control 2008;19(8):819-828.

16. Anderson BO, Braun S, Lim S and Smith RA. Early detection of breast cancer in countries with limited resources. The Breast Journal 2003; 9(2): 551-559.

17. Shirley SE, Mitchell DIG, Soares DP, James M, Escoffery CT, Rhoden AM, et al. Clinicopathologic features of Breast Diseases in Jamaica: Findings of the Jamaican Breast Disease Study 2000-2002. West Indian Med J 2008; 57 (2):90.

18. Isah RT, Sahabi SM, Muhammad AT, Okechi OO and Mohammed OM. Analysis of Female Breast Biopsies in Usman Danfodiyo University Teaching Hospital (UDUTH), Sokoto, Nigeria: A Ten year Retrospective Study. IORS Journal of Dental and Medical Sciences (IOSR-JDMS) 2013; 8(4):55-58.

19. Kumar R. A Clinicopathologic study of Breast Lumps in Bhairahwa, Nepal. Asian Pacific J Cancer Prev 2010;11:855-858.

20. Olu-Eddo $A N$ and Enoghama U. Benign Breast Lesions in an African Population: A 25-year histopathological review of 1864 cases. Nigerian Med J 2011; 52:211-216.

21. Salim SB, Nawal SB and Fabini OA. Breast diseases in Southern Yemen. Saudi Med J 2010; 31(9):1011-1014. 
22. Nuhu A, Aliyu S and Musa AB. Management of breast Lumps in Maiduguri, Nigeria. Sahel Med J 2014; 17:50-53.

23. Ellis H. Review of general surgery 1984-85. Postgraduate Med J 1985; 61:941-956.

24. Obaji NC, Elom HA, Agwu UM, Nwigwe CG, Ezeonu PO and
Umeora O. Awareness and practice of breast Self-examination among Market Women in Abakaliki, South East Nigeria. Ann Med Health Sci Res 2013; 3:7-12.

25. Umanah IN, Akhiwu W and Ojo OS. Breast tumors of Adolescents in an African Population. Afr J Paed Surg 2010; 7:78-80.

\section{Authors Contribution:}

OAU - Conceptualized the study, literature search, manuscript preparation and editing, data collection and analysis; ASNC - Designed the study, data analysis, manuscript preparation and editing; NCG and IFI - Data analysis, Manuscript preparation and editing. All authors read and approved the manuscript for submission.

\section{Authors Information:}

OAU is a consultant general surgeon, Department of Surgery, Federal Teaching Hospital Abakaliki (FETHA), Ebonyi State Nigeria. ASNC is a consultant general surgeon, Nnandi Azikiwe University Teaching Hospital Nnewi and Professor of surgery, College of Medicine, Nnamdi Azikiwe University, Anambra State, Nigeria. NCG is a consultant general surgeon, Federal Teaching Hospital, Abakaliki and lecturer in surgery, Faculty of Health Sciences, Ebonyi State University, Abakaliki, Nigeria. IFI is a consultant Pathologist, FETHA and associate professor of pathology, Faculty of Health Sciences, Ebonyi State University, Abakaliki, Nigeria.

Source of Support: None decleared. Conflict of Interest: None. 